Editor's Note: These short reviews of recent JNeurosci articles, written exclusively by students or postdoctoral fellows, summarize the important findings of the paper and provide additional insight and commentary. If the authors of the highlighted article have written a response to the Journal Club, the response can be found by viewing the Journal Club at www.jneurosci.org. For more information on the format, review process, and purpose of Journal Club articles, please see http://jneurosci.org/content/ preparing-manuscript\#journalclub.

\title{
Is Broadband Gamma Activity Pathologically Synchronized to the Beta Rhythm in Parkinson's Disease?
}

\author{
-Bernadette C.M. van Wijk \\ Department of Neurology, Charité-University Medicine, 10117 Berlin, Germany and Wellcome Trust Centre for Neuroimaging at UCL, University College \\ London, London WC1N 3BG, United Kingdom \\ Review of Cole et al.
}

Neural oscillations in the beta frequency range $(13-30 \mathrm{~Hz})$ are considered essential in motor control. Modulations in beta amplitude induced by movement were described as early as 1949 (Jasper and Penfield, 1949), yet we are only starting to grasp how beta oscillations and their interplay with neural activity at other frequencies relate to fine-grained motor control in healthy individuals and to impairments in movement disorders. Current theories ascribe an antikinetic role to beta oscillations, meaning that movements are slowed or less easily initiated when the oscillation is prominent (van Wijk et al., 2012). This is illustrated by the increased beta synchronization that can be found in basal ganglia nuclei of patients with Parkinson's disease (Hammond et al., 2007). Beta amplitude in the subthalamic nucleus positively correlates with the severity of bradykinesia and rigidity symptoms (Neumann et al., 2016; van Wijk et al., 2016) and shows a characteristic decrease with dopaminergic medication or deep brain stimulation (DBS;

Received July 17, 2017; revised Aug. 25, 2017; accepted Aug. 28, 2017.

I thank Dr. Vladimir Litvak and Prof. Andrea Kühn for comments on the manuscript.

Correspondence should be addressed to Dr. Bernadette C. M. van Wijk, Department of Neurology, Movement Disorder and Neuromodulation Unit, Campus Mitte, Charité-University Medicine Berlin, Charitéplatz 1, 10117 Berlin, Germany. E-mail: vanwijk.bernadette@gmail.com.

DOI:10.1523/JNEUROSCI.2023-17.2017

Copyright $\odot 2017$ the authors $\quad 0270-6474 / 17 / 379347-03 \$ 15.00 / 0$
Kühn et al., 2006, 2008). Remarkably, beta amplitude in cortical regions appears much less affected by the disease, at least in humans. The role of cortical oscillations in the manifestation of motor impairments, therefore, remains enigmatic.

A series of recent publications has provided new insights by showing that the coupling between beta and broadband gamma activity $(50-200 \mathrm{~Hz})$ in primary motor cortex is pathological. Beta phase and gamma amplitude have been shown to be excessively synchronized via phaseamplitude coupling (PAC) in Parkinson's disease patients but not in patients with epilepsy or dystonia (de Hemptinne et al., 2013). Moreover, when DBS is switched on, the pathological coupling decreases (de Hemptinne et al., 2015). Although these findings have sparked interest in cross-frequency analysis within the field of movement disorders, they are merely observational. There are various biological and nonbiological mechanisms that may lead to the detection of significant PAC in time series (e.g., a functional coupling between two oscillating neural populations; comodulation of lowfrequency and high-frequency components due to a common input; spiking events; nonlinear properties of synaptic transmission; small nonlinear artifacts in the recording; or improper creation of surrogate time series [Aru et al., 2015]). Without further investigation, these stud- ies provide only limited insight into parkinsonian pathophysiology.

Cole et al. (2017) conducted an indepth reanalysis of the electrocorticography recordings that were acquired from the 23 patients with Parkinson's disease in the study by de Hemptinne et al. (2015). One of their aims was to determine how much of the pathological beta-gamma coupling could be explained by nonlinear features of the beta oscillation waveform. This is important because it reveals more about the origin of the coupling, and hence about the neural mechanisms of the disease and DBS treatment. Two alternative possibilities are illustrated in Figure 1. On the one hand, if PAC is independent from the beta oscillation waveform, it is likely that beta and gamma are generated by separate (functional) processes that become coupled in the context of Parkinson's disease (Fig. 1A). On the other hand, if PAC directly results from the shape of the beta oscillation waveform, this would imply that beta and gamma coemerge from a common underlying mechanism that can be described by a single nonlinear oscillator (Fig. 1B).

Cole et al. (2017) quantified the nonlinearity of the beta oscillation waveform by introducing two metrics: oscillation sharpness and steepness. Sharpness refers to how fast the signal around an oscillation peak or trough declines. The ratio between peak sharpness and trough sharp- 
A

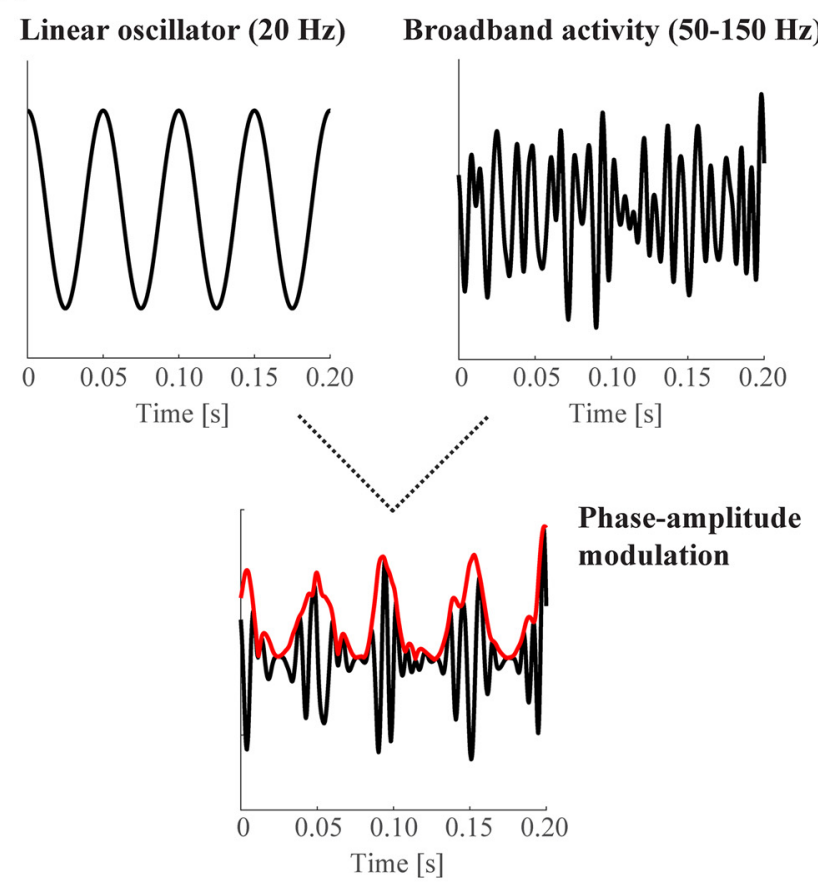

50-150 Hz amplitude / $20 \mathrm{~Hz}$ phase

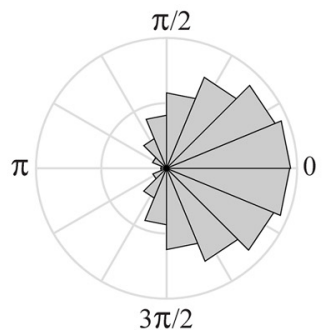

B

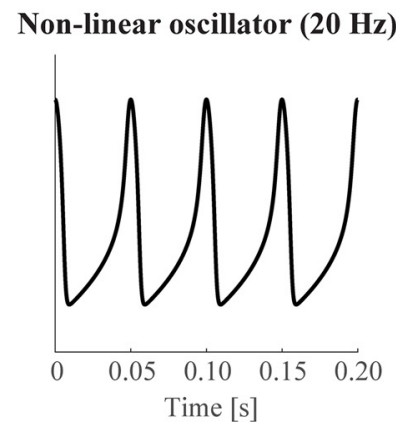

Time-frequency spectrum

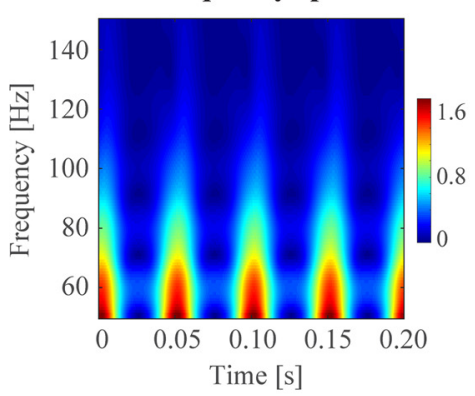

50-150 Hz amplitude / $20 \mathrm{~Hz}$ phase

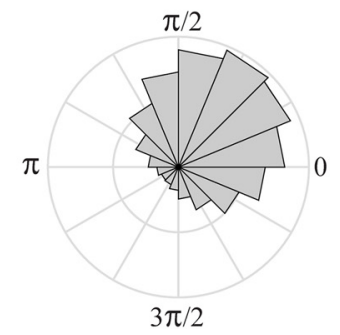

Figure 1. Simulated data illustrating two alternative mechanisms by which PAC could be generated. $A$, Two separate processes with distinct frequencies (here a linear beta oscillator and white noise bandpass filtered in the broadband gamma frequency range) become coupled in such a way that the low-frequency phase modulates the high-frequency amplitude. $\boldsymbol{B}, A$ single nonlinear oscillator produces PAC by itself. The nonsinusoidal waveform shape is reflected by a periodic increase in amplitude at harmonic frequencies. Bottom circular plots show mean $50-150 \mathrm{~Hz}$ amplitude for each $20 \mathrm{~Hz}$ phase bin (for $10 \mathrm{~s}$ of simulated data). Amplitude values are clearly modulated by phase for both mechanisms. Frequencies are chosen arbitrarily to approximately match the experimentally observed beta-gamma coupling reported in the original studies (de Hemptinne et al., 2013, 2015; Cole et al., 2017).

ness serves as a measure of waveform asymmetry. Steepness refers to how fast the waveform rises or decays within a cycle. Again, the ratio between rise steepness and decay steepness is taken as a measure of waveform asymmetry. This analysis revealed that motor cortical beta oscillations are not perfect sinusoids but form a sawtooth-like pattern (with a steep rising phase followed by a sharp peak, a slow decaying phase, and a smooth trough, or the inverse pattern; Cole et al., 2017, their Fig. 2). Moreover, DBS smoothens the oscillation to a more sinusoidal shape.

Because nonlinearities can readily produce cross-frequency coupling in time series (Aru et al., 2015), the authors investigated to what extent this was the case for the observed pathological beta-gamma PAC. Strikingly, nearly perfect and highly significant correlations were found between the sharpness ratio and average PAC strength both before DBS $\left(r=0.94, p<10^{-10}\right)$ and during DBS $\left(r=0.89, p<10^{-7}\right)$. This analysis was conducted across subjects, meaning that patients who showed strongly nonsinusoidal beta oscillations also had high PAC values. It is exceptional to find correlations this high in electrophysiological recordings, which are inherently noisy. Because PAC is a measure of coupling between beta phase and gamma amplitude, these findings clearly indicate that time-varying modulations in gamma amplitude are directly linked to nonlinear features observed in the beta oscillation waveform.

Subsequent analyses further confirmed the relationship between beta oscillation waveform and gamma amplitude. For example, gamma amplitude was high during peaks and/or troughs of the beta cycle, the gamma amplitude value at these times pos- itively correlated with the degree of sharpness of the corresponding peaks/troughs, and phase locking of gamma amplitude was stronger in beta cycles with sharper peaks/ troughs. Across subjects, gamma amplitude was also positively correlated with PAC strength. And event-related averages aligned to the onset of episodes with high gamma amplitude again revealed a sawtooth-like low-frequency pattern. All these findings are compelling evidence that beta oscillation waveform and gamma amplitude are highly interdependent.

The most likely explanation for these observations is that gamma amplitude fluctuations directly reflect the spectral components that constitute the beta oscillation waveform, as illustrated in Figure $1 B$ (see also Aru et al., 2015; Jones, 2016). This implies that no actual neural firing or synchronous neural synaptic activity 
within the gamma band needs to take place. The high-frequency components merely arise from the stereotypical nonsinusoidal shape of the beta oscillation. Hence, it is not necessary to speak of a pathological coupling between beta oscillations and neural gamma activity, which is how the original findings were primarily interpreted (de Hemptinne et al., 2013, 2015). Although this conclusion is not so explicitly stated in the article by Cole et al. (2017), it has important implications for understanding parkinsonian pathophysiology. The clinical relevance is further underscored by the disrupting effect DBS has on the beta oscillation waveform and by the significant positive correlation that was found between the sharpness ratio and patients' rigidity severity scores. Future studies should address what may lead to these abnormal nonsinusoidal beta oscillations.

In general, low-frequency components in macroscopic electrophysiological recordings are thought to reflect summed postsynaptic potentials, and high-frequency components reflect the contribution of action potentials (Buzsáki et al., 2012). A computational study showed that synchronous synaptic input from subcortical sources can create nonlinear cortical beta waveforms (Sherman et al., 2016). Such abnormal synchronous firing in the basal ganglia, including its output structures, is typically observed in the parkinsonian state (Galvan et al., 2015). Pathological subcortical activity may affect cortical function only by its impact on the timing of synaptic potentials as cortical beta and gamma power were not affected during DBS (de Hemptinne et al., 2015). The only effect observed was the altered beta oscillation waveform, leading to lower $\mathrm{PAC}$ values that are more similar to those of control subjects. It does not necessarily mean, however, that all of the observed cortical broadband gamma can be explained by the beta oscillation waveform. In fact, the distributions of gamma amplitude as a function of beta phase presented in Cole et al. (2017, their Fig. 3D-F) indicate that a substantial proportion of the gamma amplitude does not modulate with beta phase. This part of the signal may still reflect correlates of neural spiking or synaptic activity, but it is currently unclear whether it is affected by the disease.

One may be tempted to think of brain rhythms as continuously present, perfect sinusoidal oscillations. In fact, their amplitude can greatly fluctuate over time, and they can even consist of a few events that do not appear as a clear rhythm (Jones, 2016). In addition, several well studied rhythms have nonsinusoidal waveforms, including sensorimotor mu and hippocampal theta oscillations. The stereotypical shapes of these rhythms likely reflect properties of underlying physiology (Cole and Voytek, 2017). Much of this information is lost when computing spectral power as an average across a time period, as is typically done in local field potential or EEG/MEG studies. Likewise, there is a prevailing implicit assumption that biologically meaningful PAC needs to be caused by two separate oscillating neural populations whose activity becomes nested (Jensen and Colgin, 2007). Although this kind of PAC is suggestive of a newly emerged function, it could also be insightful to identify an oscillation with nonlinear characteristics from a single neural population as the PAC generator. It is nevertheless essential to distinguish between the two possibilities for a meaningful physiological interpretation.

By stepping beyond conventional analysis, Cole et al. (2017) provided evidence that previously identified pathological coupling between beta and broadband gamma is unlikely to reflect alterations in neural activities at gamma frequencies. Moreover, they demonstrated that cortical beta oscillations are indeed altered in Parkinson's disease. This suggests that not only beta amplitude but also nonsinusoidal features of the beta oscillation waveform might have an antikinetic role. Clinically, waveform sharpness might even be a promising data feature for improving closedloop DBS (Little et al., 2013) by triggering stimulation during time periods when nonsinusoidal oscillations are present. These insights make the original PAC findings (de Hemptinne et al., 2013, 2015) even more interesting and relevant. A further focus on time-varying, nonlinear data features will be crucial for advancing the field of motor control and beyond.

\section{References}

Aru J, Aru J, Priesemann V, Wibral M, Lana L, Pipa G, Singer W, Vicente R (2015) Untangling cross-frequency coupling in neuroscience. Curr Opin Neurobiol 31:51-61. CrossRef Medline

Buzsáki G, Anastassiou CA, Koch C (2012) The origin of extracellular fields and currentsEEG, ECoG, LFP and spikes. Nat Rev Neurosci 13:407-420. CrossRef Medline

Cole SR, Voytek B (2017) Brain oscillations and the importance of waveform shape. Trends Cogn Sci 21:137-149. CrossRef Medline

Cole SR, van der Meij R, Peterson EJ, de Hemptinne C, Starr PA, Voytek B (2017) Nonsinusoidal beta oscillations reflect cortical pathophysiology in Parkinson's disease. J Neurosci 37:48304840. CrossRef Medline

de Hemptinne C, Ryapolova-Webb ES, Air EL, Garcia PA, Miller KJ, Ojemann JG, Ostrem JL, Galifianakis NB, Starr PA (2013) Exaggerated phase-amplitude coupling in the primary motor cortex in Parkinson disease. Proc Natl Acad Sci U S A 110:4780-4785. CrossRef Medline

de Hemptinne C, Swann NC, Ostrem JL, Ryapolova-Webb ES, San Luciano M, Galifianakis NB, Starr PA (2015) Therapeutic deep brain stimulation reduces cortical phaseamplitude coupling in Parkinson's disease. Nat Neurosci 18:779-786. CrossRef Medline

Galvan A, Devergnas A, Wichmann T (2015) Alterations in neuronal activity in basal gangliathalamocortical circuits in the parkinsonian state. Front Neuroanat 9:5. CrossRef Medline

Hammond C, Bergman H, Brown P (2007) Pathological synchronization in Parkinson's disease: networks, models and treatments. Trends Neurosci 30:357-364. CrossRef Medline

Jasper H, Penfield W (1949) Electrocorticograms in man: effect of voluntary movement upon the electrical activity of the precentral gyrus. Arch Psychiatr Nervenkr 183:163-174. CrossRef

Jensen O, Colgin LL (2007) Cross-frequency coupling between neuronal oscillations. Trends Cogn Sci 11:267-269. CrossRef Medline

Jones SR (2016) When brain rhythms aren't rhythmic: implication for their mechanisms and meaning. Curr Opin Neurobiol 40:7280. CrossRef Medline

Kühn AA, Kupsch A, Schneider GH, Brown P (2006) Reduction in subthalamic $8-35 \mathrm{~Hz}$ oscillatory activity correlates with clinical improvement in Parkinson's disease. Eur J Neurosci 23:1956-1960. CrossRef Medline

Kühn AA, Kempf F, Brücke C, Gaynor Doyle L, Martinez-Torres I, Pogosyan A, Trottenberg T, Kupsch A, Schneider GH, Hariz MI, Vandenberghe W, Nuttin B, Brown P (2008) High-frequency stimulation of the subthalamic nucleus suppresses oscillatory activity in patients with Parkinson's disease in parallel with improvement in motor performance. J Neurosci 28:6165-6173. CrossRef Medline

Little S, Pogosyan A, Neal S, Zavala B, Zrinzo L, Hariz M, Foltynie T, Limousin P, Ashkan K, FitzGerald J, Green AL, Aziz TZ, Brown P (2013) Adaptive deep brain stimulation in advanced Parkinson disease. Ann Neurol 74: 449-457. CrossRef Medline

Neumann WJ, Degen K, Schneider GH, Brücke C, Huebl J, Brown P, Kühn AA (2016) Subthalamic synchronized oscillatory activity correlates with motor impairment in patients with Parkinson's disease. Mov Disord 31:17481751. CrossRef Medline

Sherman MA, Lee S, Law R, Haegens S, Thorn CA, Hämäläinen MS, Moore CI, Jones SR (2016) Neural mechanisms of transient neocortical beta rhythms: converging evidence from humans, computational modeling, monkeys, and mice. Proc Natl Acad Sci U S A 113: E4885-E4894. CrossRef Medline

van Wijk BC, Beek PJ, Daffertshofer A (2012) Neural synchrony within the motor system: what have we learned so far? Front Hum Neurosci 6:252. CrossRef Medline

van Wijk BC, Beudel M, Jha A, Oswal A, Foltynie T, Hariz MI, Limousin P, Zrinzo L, Aziz TZ, Green AL, Brown P, Litvak V (2016) Subthalamic nucleus phase-amplitude coupling correlates with motor impairment in Parkinson's disease. Clin Neurophysiol 127:2010-2019. CrossRef Medline 